Meta

Journal des traducteurs

Translators' Journal

\title{
Le miroir aux alouettes
}

\section{Norbert Kalfon}

Volume 34, numéro 3, septembre 1989

1. Actes du Colloque Les terminologies spécialisées: Approches quantitative et logico-sémantique et 2 . Actes du Colloque Terminologie et Industries de la langue

URI : https://id.erudit.org/iderudit/003281ar

DOI : https://doi.org/10.7202/003281ar

Aller au sommaire du numéro

\section{Éditeur(s)}

Les Presses de l'Université de Montréal

ISSN

0026-0452 (imprimé)

1492-1421 (numérique)

Découvrir la revue

Citer cet article

Kalfon, N. (1989). Le miroir aux alouettes. Meta, 34(3), 605-610.

https://doi.org/10.7202/003281ar

\section{Résumé de l'article}

Je tiens à souligner tout d'abord que mon texte fait référence au concept d'industries de la langue et non pas directement au titre de cette journée "Terminologie et marché des langues ». Je voudrais également, avant que nous ne nous mirions dans ce miroir, vous demander à tous pardon de rompre en quelque sorte le fil conducteur hautement technique de ces journées. Par ailleurs, les interventions précédentes m'ont amené à modifier la structure de mon exposé afin de commencer par vous parler de la traduction, de la terminologie et du marché des langues car, à mon sens, il est bon de présenter le point de vue d'une société qui se consacre quotidiennement à des travaux de terminologie et de traduction considérables. Enfin, je voudrais en exergue à mon exposé, vous citer deux phrases de Joseph Conrad qui dans son ouvrage Le miroir de la mer, (encore une fois un miroir), fait référence à la terminologie : " Prendre des libertés avec le langage technique est un crime contre la clarté, la précision et la beauté du parler le plus perfectionné... » Il ajoute également " ...le langage technique est un instrument poli jusqu'à la perfection par des siècles d'expérience; il est pour ce à quoi il est destiné, sans tache..."
Ce document est protégé par la loi sur le droit d'auteur. L’utilisation des services d’Érudit (y compris la reproduction) est assujettie à sa politique d'utilisation que vous pouvez consulter en ligne.

https://apropos.erudit.org/fr/usagers/politique-dutilisation/ 


\title{
LE MIROIR AUX ALOUETTES
}

\author{
NORBERT KALFON \\ Responsable du Service Terminologie \\ LOGOMOTIV \\ Espagne
}

RÉSUMÉ

Je tiens à souligner tout d'abord que mon texte fait référence au concept d'industries de la langue et non pas directement au titre de cette journée «Terminologie et marché des langues». Je voudrais également, avant que nous ne nous mirions dans ce miroir, vous demander à tous pardon de rompre en quelque sorte le fil conducteur hautement technique de ces journées.

Par ailleurs, les interventions précédentes m'ont amené à modifier la structure de mon exposé afin de commencer par vous parler de la traduction, de la terminologie et du marché des langues car, à mon sens, il est bon de présenter le point de vue d'une société qui se consacre quotidiennement à des travaux de terminologie et de traduction considérables.

Enfin, je voudrais en exergue à mon exposé, vous citer deux phrases de Joseph Conrad qui dans son ouvrage Le miroir de la mer, (encore une fois un miroir), fait référence à la terminologie :

«Prendre des libertés avec le langage technique est un crime contre la clarté, la précision et la beauté du parler le plus perfectionné...»

Il ajoute également «...le langage technique est un instrument poli jusqu'à la perfection par des siècles d'expérience; il est pour ce à quoi il est destiné, sans tache...»

S'il est un mot à la mode aujourd'hui c'est bien celui de «communication». Les gouvernements, les entreprises sont obsédés par ce terme et tout, dans leurs comportements, leurs actes, leurs décisions, n'a d'autre objectif que de soigner, développer ou améliorer la Communication. Vague et vaste, son champ sémantique ne fait que s'agrandir, donnant l'impression que tout en ce monde est régi par ce mot. De grands projets sont élaborés dans les organismes internationaux, les entreprises, les gouvernements pour faciliter les échanges, améliorer le commerce, encourager le rapprochement entre les pays ou les individus. Or, souvent, l'élément essentiel de toute «bonne communication», est laissé pour compte: la langue. Mis à part les régions du monde qui voient peser une menace sur leur langue, aucun pays n'a, pour l'instant, ressenti réellement le besoin de se doter d'une politique linguistique cohérente, par aveuglement ou par négligence. Les enjeux en la matière sont fabuleux et passionnants. Les retombées économiques d'une politique linguistique ferme et dynamique peuvent être considérables à tel point qu'on en est à parler d'industrie de la langue, de «linguismatique» comme le disait J.-C. Boulanger, d'«ingéniérie linguistique», de «terminomatique», de «documentique», de «taoïsme», etc. Derrière ces termes mystérieux se cache néanmoins une étonnante réalité : des milliers de personnes de par le monde vivent de cette industrie dont la croissance annuelle en termes de C.A. doit être bien supérieure à 50\%. (Pour compléter le chiffre que donnait P. Auger, disons que d'ici à 1992, environ 20000 postes de linguistes vont être créés en Europe, du simple fait de l'implantation du marché unique). Des budgets considérables sont dépensés 
chaque année par les entreprises en «documentation». Certaines allant même jusqu'à consacrer plusieurs millions de francs/an, qu'elles peuvent rarement épuiser, faute d'entreprises spécialisées capables de répondre à leur demande.

À l'opposé, Boeing ou Airbus par exemple, rechignent à faire traduire la documentation de leurs appareils, étant donné qu'il faudrait vendre à chaque pays acquéreur un ou deux appareils de plus pour couvrir les coûts de rédaction et de traduction de cette documentation! D'où l'énorme intérêt que suscite la traduction automatique et la non moins énorme déception qui en découle très souvent.

Depuis 1947, des millions de dollars ont été et sont investis en recherche et développement dans le secteur de la T.A. et aucune application industrielle tangible n'en a résulté, si ce n'est dans quelques secteurs isolés et très spécialisés. Quarante années d'incessantes recherches pour un résultat à priori plus que médiocre! A priori, car d'autres «produits» de ces recherches ont pu être exploités et ont apporté des solutions, souvent décisives, à de nombreux utilisateurs.

La terminologie est, peut être, la discipline qui a permis les progrès les plus significatifs en la matière. En réalité, pour grand nombre de linguistes, il était plus urgent d'essayer d'automatiser ce qui pouvait l'être, plutôt que de s'acharner à trouver une réponse informatique universelle en matière de traduction. D'ailleurs les concepteurs /développeurs de systèmes de T.A. font le chemin à l'envers car les seuls débouchés économiques dans ce domaine se trouvent dans les secteurs industriels et de services, pour lesquels des millions de termes sont nécessaires. D'où également la prolifération de fabricants de terminologie dans certains pays. On pourrait citer les recherches intenses menées au Japon, recherches encore une fois résolument soutenues par le gouvernement japonais qui en a décidément bien compris les enjeux politiques et surtout économiques.

À l'extrême, il est permis de penser que d'ici quelques années, il sera possible de trouver un excellent dictionnaire d'agriculture en espagnol... élaboré et édité au Japon. Pourquoi pas? Qui oserait en faire le reproche aux japonais, alors qu'aucune entreprise, qu'aucun organisme espagnol n'aura été capable de proposer la même chose? Les secteurs d'activité concernés par les produits terminologiques sont multiples : l'édition bien sûr, mais aussi les services de consultation de Banques de Données, le vidéodisque, le CD. ROM, les éditeurs de logiciels, les développeurs de systèmes de T.A.O., pour n'en citer que quelques-uns. Et ne parlons pas des répercussions économiques que peut représenter le fait de mettre sur les marchés mondiaux, avant les autres, un produit de consommation accompagné d'une documentation (bien) traduite dans toutes les langues majeures.

Alors après cette introduction je dois dire que je me réjouis depuis quelques années de voir à quel point ma profession occupe de plus en plus une place prépondérante dans le monde d'aujourd'hui.

Le nombre impressionant de colloques et de rencontres qui ont lieu dans les domaines traités au cours de ces deux journées le prouvent amplement. Même si le monde des affaires ne voit pas encore clairement ce type de profession comme nous tous ici. Je reviendrai plus en détail sur ce que j'ai appelé «ma profession» et sur ce qu'elle représente de fait.

En dépit de ma timidité maladive, je souhaiterai vous raconter mon expérience personnelle dans les domaines de la traduction et de la terminologie (je serai très bref, ne vous inquiétez pas). Je la résumerai ainsi : douze ans d'enfer et, pour l'instant, deux ans de purgatoire en attendant le paradis que les industries de la langue ne manqueront pas de nous offrir très bientôt, si j'en juge par le nombre extraordinaire de projets, de recherches, d'études et de produits qui existent aujourd'hui et dont certains nous ont été présentés à ce colloque. En ce qui concerne ces douze années d'enfer, je dirai qu'elles ont représenté 
pour une grande part ma période de formation sur le tas, très courte comme vous pouvez le constater, et qu'elles m'ont prouvé au moins deux choses : l'extrême solitude du traducteur et ... la solitude extrême du terminologue face aux travaux qui leur sont confiés. Le traducteur des années 1980 ressemble encore fortement au premier traducteur de la Bible, même s'il a remplacé un jour sa plume par une machine à écrire et plus tard, peutêtre, par un ordinateur. Aucun bouleversement significatif n'est apparu dans le milieu traduisant depuis des millénaires.

Combien de traducteurs, ici présents, ont informatisé leur processus de travail? Combien de traducteurs savent ce qu'est la terminologie et savent «en faire»? Quelle intervention au cours de ce colloque a fait référence aux problèmes des méthodes de travail et de méthodologie générale dans le secteur des prestations de services linguistiques? Combien d'entreprises au monde posent correctement les problèmes de gestion de la documentation mono ou multilingue? Quelle école propose aux futurs traducteurs une formation complète sur ce qu'est ou va être l'industrie de la langue et sur les besoins considérables du marché européen de l'après 1992? Quel centre de recherche en T.A. emploie à temps plein une équipe de traducteurs professionnels (et je ne dis pas de linguistes-informaticiens) pour développer le plus harmonieusement possible ces fabuleux outils dont on nous rebat les oreilles et dont M. Bill Forest fait les frais chaque fois que la machine traduit son nom par M. Facture Forêt?

Lamentablement nous en sommes encore là, et je défie quiconque ici d'apporter une réponse claire et complète aux questions que je viens de poser.

Alors, après avoir traduit, ou corrigé, plus de 30000 pages en douze ans, après avoir traité, d'une façon ou d'une autre, près de 50000 termes techniques en plusieurs langues, je me suis rendu à l'évidence qu'il fallait que je prenne mon destin en main. Ainsi, - et je passe directement au purgatoire - en compagnie de professionnels souhaitant rompre leur solitude et partageant totalement une vision intégrante des multiples services linguistiques, avons-nous décidé de créer une société dont l'objectif primordial concerne davantage le travail en équipe et la constante réflexion méthodologique que le montage d'une affaire qui va rapporter beaucoup d'argent.

Or, et pour rester dans ces comparaisons plus ou moins bibliques, le miracle s'est produit. Nous avons créé une société rentable. Nous avons développé nous-mêmes des outils de travail et avons constitué un réseau d'entreprises en Europe que je crois rare dans sa conception et sa philosophie.

L'objectif majeur de l'implantation de ce réseau n'est pas de lancer sur le marché européen une multinationale de prestations de services linguistiques mais bien de nous donner les moyens de la réflexion et de proposer des solutions de terrain à tous ceux qui depuis des années s'efforcent vainement de résoudre nos problèmes. C'est pourquoi en compagnie d'une entreprise espagnole et d'une entreprise portugaise, avons nous élaboré le projet PREDICAT dont je dirai qu'il est surtout, et avant tout, une réflexion d'entreprise «de traduction» sur les moyens à mettre en place et les outils à développer pour mieux appréhender l'immense marché linguistique qui s'offre à elles. Cette réflexion doit en rejoindre d'autres et doit être prise en compte impérativement par tous ceux qui veulent que les choses bougent en la matière. Car elle est le résultat de nombreuses années d'expérience et, pourquoi ne pas le dire, d'un profond désespoir.

Il me faut, par conséquent, vous parler plus en détail du projet PREDICAT. Je ne vais que vous en tracer les grandes lignes, par manque de temps et surtout parce qu'il est plus important pour l'instant de situer le contexte réel du travail des terminologues et des traducteurs.

L'axe principal de ce projet concerne la formation de professionnels de la langue à la réalité industrielle et économique des travaux que nous menons. Formation destinée à 
des post-universitaires et qui va de l'informatique à la gestion de la documentation en insistant sur des travaux en temps réel, la terminologie, la P.A.O., etc.

L'autre axe concerne la structure et le développement d'outils de production et méthodologiques, la fabrication de terminologies diverses et de pointe et les échanges entre nos trois sociétés et d'autres partenaires éventuels.

Je ne crois pas qu'il soit nécessaire d'en dire beaucoup plus pour le moment. La tâche, comme vous le constatez, est immense. Quoi qu'il en soit notre avantage en la matière, malgré nos faibles ressources - n'oublions pas que nous sommes de petites entreprises - est que nous n'avons pas le choix. Nous avons par ailleurs l'intime conviction que, pour l'instant, nous ne pouvons attendre grand chose de l'extérieur. La solution à nos problèmes dépend, pour une part considérable, exclusivement de nous. Et je peux conclure ce chapitre en affirmant que le simple fait au sein de ce réseau, d'avoir échangé des idées, des méthodologies et d'avoir réalisé ensemble des travaux de grande envergure, a situé, dans chaque pays, l'entreprise associée au projet PREDICAT, à une toute première place sur son marché.

J'aurais souhaité à présent annoncer la naissance d'une nouvelle profession, d'un nouveau métier : celui de spécialiste ou expert en communication multilingue. Je pourrais annoncer également la naissance des Sociétés de Services en Ingéniérie Linguistique. J'aimerais vous relater la chronique d'une mort annoncée: celle du traducteur traditionnel. Je pourrais vous décrire le profil du traducteur et du terminologue de l'an 2000 et vous convaincre, et me convaincre, qu'à ce moment-là tout baignera dans l'huile.

Mais est-ce vraiment intéressant? N'est-il pas plus important de faire le constat de la situation présente et d'essayer d'aborder les problèmes qui se posent à nous avec les moyens et les outils dont nous disposons?

Combien de sociétés ou de traducteurs se mordent les doigts d'avoir fait l'acquisition de systèmes automatiques ou semi-automatiques qui ne leur servent à rien ou à pas grand chose? Je pourrai vous citer une trentaine d'entreprises dans le monde qui ont dépensé des millions à l'achat de systèmes que personne en leur sein ne peut ou ne veut utiliser. Combien d'entreprises fournissent les textes à traduire sur support magnétique? De plus en plus certes, mais la proportion est encore minime. À quoi me servirait alors un logiciel d'extraction automatique de terminologie si je dois d'abord faire l'achat de systèmes de lecture optique onéreux et peu fiables, sans parler de cette extraction automatique qui n'en est pas une?

A quoi me servirait la connexion à la BTQ ou à Termium si les coûts d'interrogation et de communication sont extraordinairement élevés, même si l'accès à l'information est gratuit ?

À quoi me sert un outil de correction automatique, si, dès lors que je remplace un féminin par un masculin ou un singulier par un pluriel, je dois corriger manuellement les phrases aberrantes qui résultent de cette correction automatique?

Alors, lorsque je parle de miroir aux alouettes, c'est parce qu'on nous fait miroiter des solutions qui n'en sont pas et que l'on nous fait croire que les alouettes vont tomber toutes rôties dans nos écuelles. En disant cela, je ne mets pas en cause la valeur intrinsèque de ces produits ni la qualité des recherches qui ont permis d'y aboutir. Ce que je rejette avec la plus grande vigueur c'est bien souvent la malhonnêteté intellectuelle des vendeurs de ces produits qui n'en mentionnent jamais les contraintes et les limites, qui ne nous parlent pas des coûts considérables de mise en place et qui, avec quel culot, nous offrent des boîtes vides. (Imaginez que vous entriez dans un magasin de chaussures et que vous en ressortiez avec une seule chaussure ou, encore mieux, seulement avec l'emballage. Soit le vendeur est très fort, soit l'acheteur n'est pas très intelligent. C'est le moins que l'on puisse dire.) J'ose affirmer que, mis à part les logiciels de traitement de textes, 
les SGBD du type DBIII ou autres, (et maintenant Multiplan depuis l'intervention de M. Corbeil), il ne faudrait rien acheter pour l'instant car l'industrie ne peut, de toute évidence, rien nous offrir de mieux!

Car le concept d'industrie de la langue est loin d'être rempli... À moins qu'on ne se persuade que ce qui est encore au stade de la recherche est déjà industriel; qu'une banque de données terminologiques est accessible à tous; que la T.A.O. se trouve sur les bureaux de tous les traducteurs; que nos traitements de texte sont munis d'outils puissants pour indexer, extraire, corriger, réviser... Et qu'à l'instar de l'industrie de la chaussure (dont je parlais il y a un instant), de la tannerie au fabricant de lacets, tous les intervenants de la chaîne industrielle ont des objectifs convergents.

Or je pense que nous sommes encore loin de tout cela. Chacun, dans le microcosme langagier travaille dans un coin, refaisant souvent ce que d'autres ont déjà fait.

En parcourant la liste des participants à ce colloque, je m'aperçois qu'il y a des traducteurs, des terminologues, des documentalistes, des chercheurs, des informaticiens, etc.

Imaginez maintenant qu'au lieu de m'écouter parler, vous décidiez de vous réunir autour d'une table et d'échanger vos expériences; de faire part de vos besoins et de vos souhaits. Peut être qu'il en sortirait quelque chose de positif, et que commencerait à s'instaurer un vrai débat sur la nécessité d'apporter des solutions en équipe. Nous serions tous plus forts pour frapper aux portes des institutions afin d'obtenir les incitations nécessaires à l'implantation d'une industrie. Le projet LIFE de la CCE par exemple va dans ce sens et peut apporter à certains le coup de pouce dont ils ont besoin.

Toutefois je ne crois pas qu'il faille attendre grand chose de ces subventions éventuelles. Certains en vivent fort bien depuis des décennies et, à ma connaissance, rien de bien tangible n'en est ressorti.

La solution, à mon sens, pour que s'implante une véritable industrie de la langue, repose surtout sur l'iniciative privée et sur la faculté qu'auront certaines entreprises de développer leurs services internes de documentation.

En effet, leurs besoins sont considérables, des milliers de pages ne sont pas traduites faute de moyens financiers et humains ou d'entreprise capable d'absorber en soustraitance ces volumes immenses. (Et les besoins des entreprises ne sont pas exclusivement linguistiques. Il faut traiter la documentation, l'éditer, la classer. Un traitement partiel ne les intéresse pas). Il convient de leur apporter des solutions neuves et intégrantes. Il faut être capable, lorsqu'un produit doit être lancé sur le marché mondial, de proposer une diffusion multilingue de la documentation qui l'accompagne. Il est nécessaire de savoir adapter et «localiser» des programmes informatiques en plusieurs langues. Il convient d'assurer la cohérence linguistique — donc terminologique — de tous leurs services, départements et produits. Il est indispensable de savoir fabriquer cette documentation. Sans compter sur les besoins des instances européennes qui voient arriver avec préoccupation cette intégration dont on parle tant et pour laquelle - sur le plan linguistique tout au moins - rien de déterminant n'a encore été fait; si ce n'est bien entendu l'existence de la banque de données EURODICAUTOM qui à elle seule ne permettra pas d'assurer la communication multilingue tous azimuts qui découlera de l'implantation du marché unique en 1993.

Je me suis efforcé d'évoquer ici ce que je ressens au plus profond de moi-même. On pourrait déceler sous ce discours une certaine démagogie ou vision retardataire du monde de l'industrie de la langue. Il n'en est rien. Je suis convaincu que les principes et l'expérience de mon entreprise vont dans le sens d'une industrialisation et d'une automatisation quasi-complètes des processus linguistiques. Mais pas à n'importe quel prix et en refusant d'entendre le chant des sirènes linguistiques. Il est temps de tirer un trait sur l'immense gâchis humain et économique que les chantres de l'universalité en toute matiè- 
re ont entraîné. Il n'existe aucune solution universelle et totalitaire à nos problèmes d'échanges et de communication linguistiques.

Alors dans le miroir aux alouettes dont je viens de parler je vous demande simplement de projeter trois lettres «T.A.O.», à l'envers dans ce miroir vous verrez «O.A.T.» Outils d'aide à la traduction ou d'aide à la terminologie. Des centaines, des milliers d'outils différents et complémentaires peuvent être créés - et il en existe déjà beaucoup - nous en choisirons les meilleurs: $70 \%$ des travaux que nous menons peuvent être parfaitement automatisés (terminologie, préparation de textes, correction stylistique et orthographique, édition, etc.). Les $30 \%$ restants font appel à notre acquis, à notre savoir-faire et à notre génie linguistique. Et peut-être qu'ainsi M. Bill Forest ne s'arrachera plus les cheveux en découvrant qu'il s'appelle en français M. Facture Forêt. 